\title{
CTLA-4-mediated regulatory phenotype of T-cells in tolerant lung recipients
}

\author{
K. Botturi*, Y. Lacoeuille*, P. Thomas*, S. Boniface*, \\ M. Reynaud-Gaubert* and A. Magnan*
}

ABSTRACT: Obliterative bronchiolitis $(O B)$ is the major cause of long-term lung allograft loss resulting from an unclear immune process occurring in the absence of the donor's immune cells. The present authors hypothesised that interactions of autologous dendritic cells (DCs) with Tcells could differ in $O B$ patients compared with healthy lung transplant recipients (LTRs).

Monocyte-derived DCs from 14 OB and 35 non-OB LTRs were cultured with autologous T-cells. T-regulatory (Treg) cells, co-receptors, cytokine production, DC phenotype and indoleamine 2,3dioxygenase (IDO) expression were assessed by flow cytometry. Experiments were repeated in the presence of Pseudomonas aeruginosa or anti-co-receptor antibodies.

DCs from non-OB LTR upregulated Treg cells, cytotoxic T-lymphocyte antigen (CTLA)-4 and interleukin (IL)-10. By contrast CD28 and inducible T-cell co-stimulator were downregulated concomitantly to IL-13 and IL-4. Compared to OB, non-OB DCs displayed an immature phenotype with lower CD80 and CD83 and higher IDO levels of expression. Stimulation by $P$. aeruginosa did not abolish the tolerogenic effects of DCs on non-OB T-cells. Finally, decreased Treg cells and IL10 production were detected when adding anti-CTLA-4 antibodies in non-OB LTR.

The present study demonstrates that dendritic cells from nonobliterative bronchiolitis lung transplant recipients induce a tolerant T-cell phenotype which is dependent on cytotoxic T-lymphocyte antigen-4 engagement.

KEYWORDS: Cytotoxic T-lymphocyte antigen-4, dendritic cells, lung transplantation, obliterative bronchiolitis, T-regulatory cells

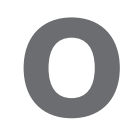

bliterative bronchiolitis $(\mathrm{OB})$ is the manifestation of chronic lung allograft rejection occurring in $33 \%$ of patients 5 yrs after surgery [1]. Despite a clear decrease in the prevalence of the complication compared with earlier studies, bronchiolitis obliterans syndrome (BOS) remains by far the most significant long-term complication and the first cause of late post-operative death, accounting for $25-30 \%$ of all deaths occurring beyond the third year after surgery [1]. In addition, BOS also causes significant loss of health-related quality of life [2]. At the histological level, OB is characterised at the early stage by the infiltration of the bronchiolar wall with lymphocytes, monocytes and histiocytes, followed by a fibrosis process, leading to obliteration of small airways [3]. Although the understanding of risk factors associated with the occurrence of $\mathrm{OB}$ has increased in the recent years [4], immune characteristics distinguishing tolerant recipients from patients with BOS remain largely unknown $[5,6]$.
Acute rejection (AR) episodes are recognised as important risk factors for OB. AR mainly occurs within the first post-operative months, when donors' leukocytes and antigen presenting cells (APCs) are still infiltrating the bronchi, so that a classical alloreactive immune response is incriminated. Dendritic cells (DCs) are the most potent APCs in the lung, interacting with T-cells in association with major histocompatibility complex (MHC) class-II molecules. T-cells specifically recognise alloreactive MHC via T-cell receptor (TCR). In addition to the cognate TCR-MHC recognition, engagement of co-receptors expressed on APC and T-cells is required for the occurrence of a productive immune response. These co-stimulatory signals are mediated via CD80/CD86 molecules (B7-1/B7-2) on DCs and CD28 or cytotoxic Tlymphocyte antigen (CTLA)-4 receptors on T-cells, leading to positive or negative regulation of T-cell activation, respectively. A recent study on gene expression profile in bronchoalveolar lavage (BAL) showed that both CD28 and CTLA-4 co-receptors were upregulated during AR [7].
AFFILIATIONS

*Faculty of Medicine, UPRES-EA 3287, University of the Mediterranean, and \#IFR Jean Roche, EA 2201, Marseille, France.

CORRESPONDENCE

K. Botturi

L'Institute du Thorax Equipe AVENIR-INSERM U915 Faculté de Médecine 1 rue Gaston Veil 44035 Nantes Cedex 1 France

Fax: 33240412950

E-mail: botturikarine@yahoo.fr

Received:

July 232007

Accepted after revision:

January 212008

SUPPORT STATEMENT

This work was supported by the Association Vaincre La Mucoviscidose, Paris, France.

STATEMENT OF INTEREST None declared. 


\begin{tabular}{|c|c|c|}
\hline & Non-OB & OB \\
\hline Subjects & 35 & 14 \\
\hline Age yrs & $39 \pm 2.01$ & $34.86 \pm 3.52$ \\
\hline \multicolumn{3}{|l|}{ Sex } \\
\hline Male & 20 & 4 \\
\hline Female & 15 & 10 \\
\hline \multicolumn{3}{|l|}{ Transplantation } \\
\hline Single & 3 & 0 \\
\hline Bilateral & 32 & 14 \\
\hline \multicolumn{3}{|l|}{ Indication } \\
\hline Cystic fibrosis & 23 & 8 \\
\hline Emphysema $\alpha 1$-antitrypsin & 7 & 1 \\
\hline Bronchiectasis & 1 & 1 \\
\hline Fibrosis & 1 & 1 \\
\hline Primary pulmonary hypertension & 0 & 2 \\
\hline Others & 2 & 1 \\
\hline FEV $1 \%$ pred & $84.29 \pm 3.22$ & $38.07 \pm 3.53$ \\
\hline Months after transplantation & $190.57 \pm 143.42$ & $88.28 \pm 15.54$ \\
\hline \multicolumn{3}{|l|}{ HLA-A, HLA-B, HLA-DR mismatch } \\
\hline $0-2$ & 0 & 0 \\
\hline $3-4$ & 2 & 1 \\
\hline $5-6$ & 33 & 13 \\
\hline $\begin{array}{l}\text { Cytomegalovirus mismatch } \\
\text { (donor }+ \text { / recipient -) }\end{array}$ & 3 & 2 \\
\hline \multicolumn{3}{|l|}{ Treatment (if sampled) } \\
\hline TAC, AZA & 7 & 0 \\
\hline CsA, AZA & 10 & 0 \\
\hline TAC, MMF & 5 & 0 \\
\hline CsA, MMF & 1 & 0 \\
\hline TAC, AZA, AZI & 2 & 4 \\
\hline TAC, MMF, AZI & 10 & 10 \\
\hline \multicolumn{3}{|l|}{ Pseudomonas aeruginosa } \\
\hline Pre-transplant colonisation & 24 & 8 \\
\hline Post-transplant re-colonisation & 19 & 8 \\
\hline
\end{tabular}

Data are expressed as $\mathrm{n}$ or mean \pm SE. OB: obliterative bronchiolitis; FEV1: forced expiratory volume in one second; \% pred: \% predicted; HLA: human leukocyte antigen; TAC: tacrolimus; AZA: azathioprine; CsA: cyclosporine; MMF: mycophenolate mofetil; AZI: azithromycin.

$\mathrm{OB}$ is an inflammatory process which occurs at distance to the procedure, when donors' cells, but not bone marrow cells, are locally renewed and proliferating [8, 9]. Previous studies showed that an increased number of DCs were present in chronically rejecting lung allografts compared with stable lung transplant recipients (LTR) [10, 11]. It has previously been demonstrated that T-cells were activated during chronic rejection, with deficient T-regulatory (Treg) cell activation [12]. Thus, OB could result from a DC/T-cell interaction in which both DC and T-cells are recipient's cells. This interaction could become an antigenic presentation in which the presented peptide, likely a donor-derived antigen, would bear the alloreactive specificity. The present authors hypothesised that, dependent upon the nature of DC/T-cell interaction in terms of $\mathrm{T}$-cell activation or tolerance induction, either $\mathrm{OB}$ or graft tolerance could occur, respectively, irrespective of the presented peptide.

First, in order to test this hypothesis, the interactions of autologous monocyte-derived DCs with T-cells were investigated in LTR. T-cell activation in DC/T-cell co-cultures between healthy lung transplant recipients (non-OB) and recipients with BOS were compared. Secondly, as infection is a factor potentially associated with $\mathrm{OB}$, this interaction was also tested in the presence of microbial compounds. Finally, the role of co-receptors was analysed in this process.

\section{MATERIAL AND METHODS Study design}

Blood samples were collected in consecutive LTR from July 2005 to April 2007. The study was approved by the Sud-Méditerannée II Ethic Committee (Marseille, France) and written informed consent was obtained from all the patients. In order to avoid bias related to perioperative complications, LTRs within 6 months of transplant were not included. Microbiological examination was systematically performed in BAL samples and those with relevant infection (positive microbiology with clinical or radiological findings requiring specific antimicrobial therapy) were excluded from the study. In addition, samples taken within the 2 months following the treatment of infection or acute rejection were not considered.

BOS was diagnosed on a progressive bronchial obstruction according to previous guidelines [13]. Details on follow-up, respiratory function and additional treatments of medication dosages are given in the online supplement. BOS-0p recipients received an increase in immunosuppressive regimen, especially in steroids and so, to avoid bias linked to treatment, these recipients were excluded from the study.

\section{DC differentiation and co-culture with autologous T-cells}

Peripheral blood mononuclear cells (PBMCs) were isolated from peripheral blood by Ficoll-Hypaque gradient-centrifugation. Monocytes were purified from PBMCs by adherence and differentiated into DCs, as previously described [14]. Briefly, monocytes were cultured in 24 -well plates $\left(10^{7}\right.$ cells $\left.\cdot \mathrm{mL}^{-1}\right)$ for $24 \mathrm{~h}$ in complete medium supplemented with $800 \mathrm{U} \cdot \mathrm{mL}^{-1}$ granulocyte-macrophage colony-stimulating factor (GM-CSF) and $500 \mathrm{U} \cdot \mathrm{ml}^{-1}$ interleukin (IL)-4. Cells were then incubated with a combination of pro-inflammatory mediators for another $24 \mathrm{~h}\left(1000 \mathrm{U} \cdot \mathrm{mL}^{-1}\right.$ tumour necrosis factor- $\alpha, 10 \mathrm{ng} \cdot \mathrm{mL}^{-1} \mathrm{IL}-1 \beta$, $10 \mathrm{ng} \cdot \mathrm{mL}^{-1}$ IL-6 and $1 \mu \mathrm{M}$ prostaglandin-E2). Autologous lymphocytes, recovered from nonadherent cells, were kept in fresh medium for the duration.

On day 2, monocyte-derived DCs were then co-cultured with autologous lymphocytes at a ratio of $1: 10$ for 5 days in presence of IL-2 and IL-7. On day 7, cells were harvested for staining. In some experiments, the ratio of DC on T-cell was increased from 1:10 to $4: 10$.

\section{Bacterial stimulation}

To assess the characteristics of DC/T-cell interactions in the presence of microbial compounds, lysed Pseudomonas aeruginosa was added to co-cultures. P. aeruginosa was chosen as a source of recall antigens for the majority of patients displaying 

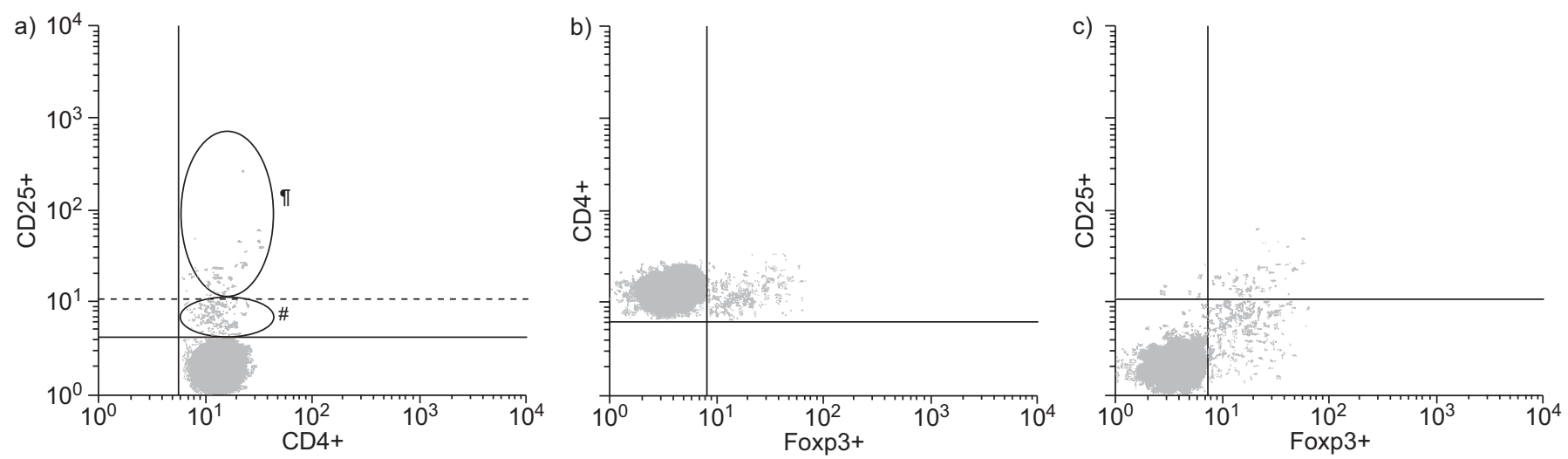

FIGURE 1. T-cell phenotype in dendritic cell/T-cell co-cultures. T-regulatory cells (CD4+CD25+Foxp3+) were assessed by flow cytometry. a) CD25+ versus CD4+ intensity; b) CD4+ versus Fox3p+ intensity; and c) CD25+ versus Fox3p+ intensity, $2.3 \%$ were positive for both. ${ }^{\#}$ : CD4+CD25medium+ cells; ": CD4+CD25high+ cells. Collectively CD4+CD25medium+ and CD4+CD25high+ represent all CD4+CD25+ cells.

cystic fibrosis (CF) or bronchectasis. DCs were incubated with $10 \mu \mathrm{L}$ of $P$. aeruginosa culture supernatant on day 2 . After $12 \mathrm{~h}$ of incubation, DCs were co-cultured with autologous lymphocytes.

\section{Co-receptor blockade}

To determine the role of co-receptors in DC/T-cell interactions, cultures were performed from day 2 with or without anti-CD28 (clone CD28.6, $10 \mu \mathrm{g} \cdot \mathrm{mL}^{-1}$ ), anti-inducible T-cell co-stimulator
(anti-ICOS; clone ISA-3, $20 \mu \mathrm{g} \cdot \mathrm{mL}^{-1}$ ) or anti-CTLA-4 (clone 14D3, $20 \mu \mathrm{g} \cdot \mathrm{mL}^{-1}$ ) monoclonal antibodies (Abs; all from eBioscience, San Diego, CA, USA) [15].

\section{Flow cytometry}

Expression of T-cell membrane antigens was assessed by adding specific Abs to cells at recommended concentrations (CD4-PECy5, CD25-fluorescein isothiocyanate (FITC); Beckman Coulter, Marseille, France; CD3-FITC; Dako, Trappes, France;
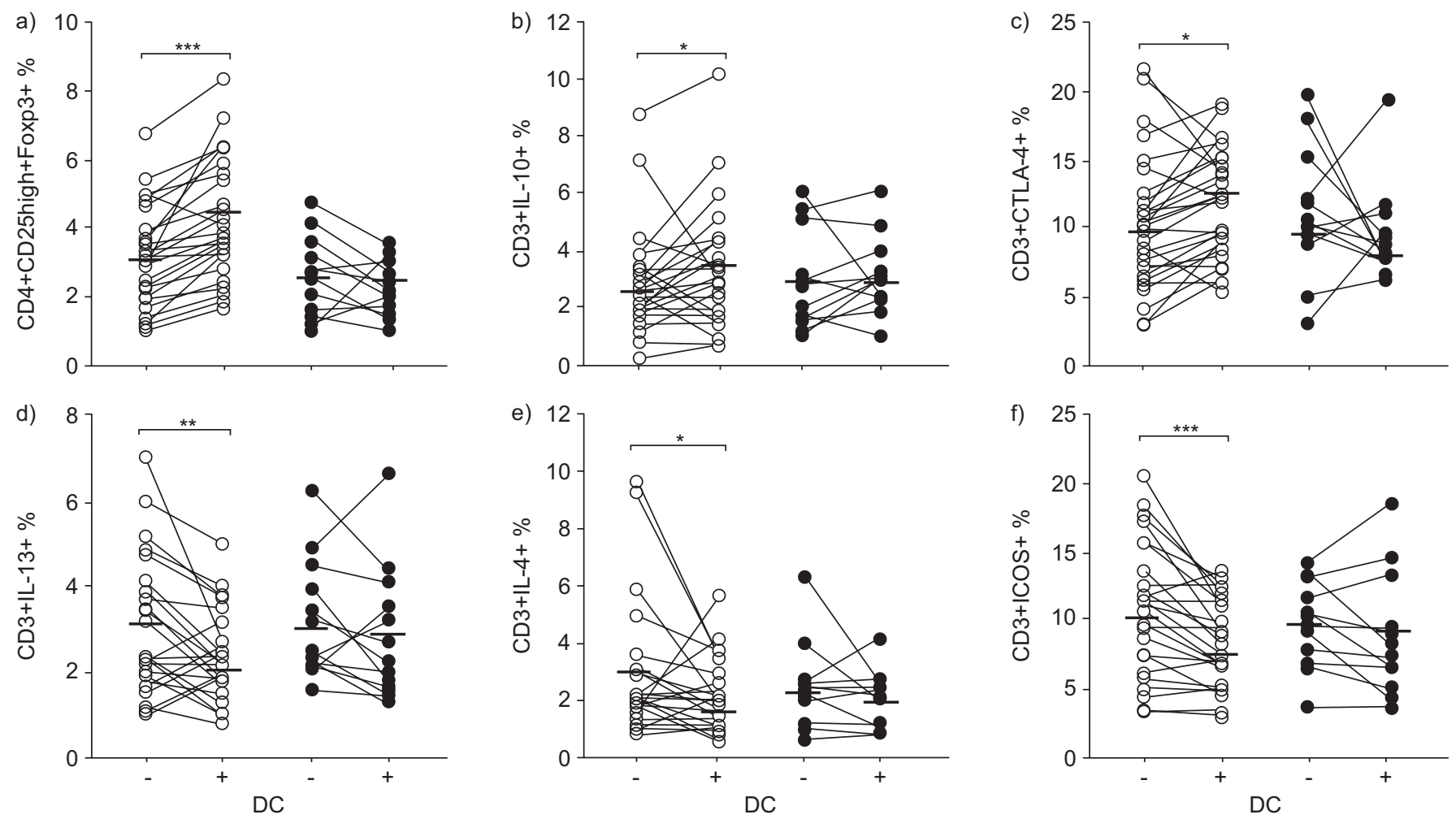

FIGURE 2. Cytokine production in dendritic cell/T-cell co-cultures. Interleukin (IL)-13, IL-4 or IL-10 producing T-cells as well as T-regulatory cells (CD4+CD25high+Foxp3+), inducible T-cell co-stimulator (ICOS) and cytotoxic T-lymphocyte antigen (CTLA)-4 expressing T-cells were assessed in obliterative bronchiolitis $(\mathrm{OB} ; \boldsymbol{\bullet} ; \mathrm{n}=14)$ and non-OB $(\mathrm{O} ; \mathrm{n}=30)$ recipients, in the presence $(+)$ or absence $(-)$ of monocyte-derived denditic cells (DC). Results were expressed as percentage of total T-cells. Horizontal bar shows mean. *: $p<0.05 ; * *$ : $p<0.01$; ***: $p<0.001$. 


\begin{tabular}{|c|c|c|c|c|}
\hline \multirow[t]{3}{*}{ TABLE 2} & \multicolumn{4}{|c|}{$\begin{array}{l}\text { Effect of dendritic cells on T-cell surface antigens } \\
\text { and interferon (IFN)- } \gamma \text { expression in obliterative } \\
\text { bronchiolitis (OB) and non-OB recipients }\end{array}$} \\
\hline & \multicolumn{2}{|c|}{ Non-OB } & \multicolumn{2}{|c|}{ OB } \\
\hline & $-D C$ & $+\mathrm{DC}$ & $-D C$ & $+\mathrm{DC}$ \\
\hline Trials $\mathbf{n}$ & 30 & 30 & 14 & 14 \\
\hline CD3+IFN- $\gamma+$ & $11.65 \pm 1.41$ & $11.89 \pm 1.51$ & $14.52 \pm 2.54$ & $18.11 \pm 2.51^{*}$ \\
\hline $\mathrm{CD} 3+\mathrm{CD} 69+$ & $16.78 \pm 1.36$ & $14.05 \pm 1.13^{\star \star}$ & $14.59 \pm 1.93$ & $15.27 \pm 2.39$ \\
\hline CD3+CD28+ & $81.14 \pm 2.17$ & $75.94 \pm 2.35^{\star \star \star}$ & $80.35 \pm 4.72$ & $75.07 \pm 4.48^{*}$ \\
\hline
\end{tabular}

Data are presented as mean \pm SE of total T-cell, unless otherwise stated. T-cells from non- $\mathrm{OB}$ and $\mathrm{OB}$ recipients were cultured in the presence (+) or absence (-) of autologous monocyte-derived dendritic cells (DC). T-cell phenotype was assessed by flow cytometry. Statistics are compared within each group (non$\mathrm{OB}$ or $\mathrm{OB}$ ), IFN- $\gamma$ and receptors expression in the presence or absence of $\mathrm{DC}$. $*: p<0.05 ; * *: p<0.01 ; * * *: p<0.001$.

CD3- PECy5, CD69-PE; Immunotools, Friesoythe, Germany; CD28-FITC, ICOS-PE, CTLA4-PE-Cy5; BD Bioscience, Le Pontde-Claix, France). Intracellular foxp3 expression by CD4+ T-cells was measured after cell fixation and permeabilisation,
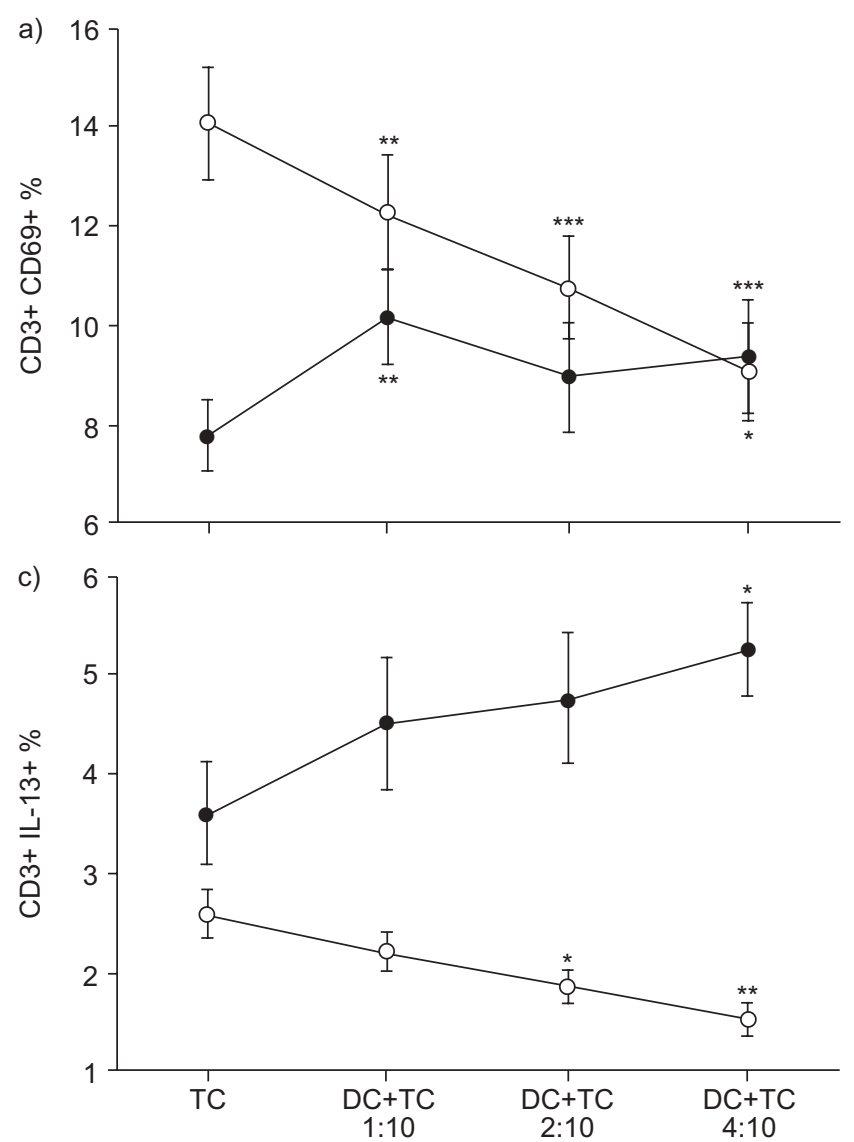

by staining with intracellular antihuman Foxp3-PE antibodies (eBiosocience staining kit). T-cell cytokine production was also measured after cell fixation and permeabilisation by staining T-cells with intracellular Abs (interferon (IFN) $\gamma$-FITC, IL-4-FITC; BD Bioscience; IL13-PE, IL-10-PE; R\&D system, Lilles, France) after a 6-h incubation in presence of monensin, as previously described [16]. Expression of DC membrane antigens was studied [17] using specific Abs at recommended concentrations (CD83-PE, CD11c-PE, human leukocyte antigen (HLA)-DR-PC5; Beckman Coulter; CD80FITC; Immunotools).

DC indoleamine 2,3-dioxygenase (IDO) was also assessed by intracellular staining with rabbit antihuman IDO Abs (Chemicon, St-Quentin-en-Yvelines, France), counterstained by FITC-labeled swine antirabbit secondary Abs (Dako).

Fluorescence was detected with a $15 \mathrm{~mW}$ argon ion laser on a three colors FACSCan ${ }_{\Re}$ flow cytometer (Becton Dickinson, Franklin Lakes, NJ, USA). Standard acquisition and analysis software were obtained through Cellquest ${ }_{\circledR}$ Software (Becton Dickinson).

\section{Statistical analysis}

Normal distributions of the variables were assessed by negativity of a Kolmogorov-Smirnov test. Comparisons between groups were determined using t-tests. Paired t-tests were used
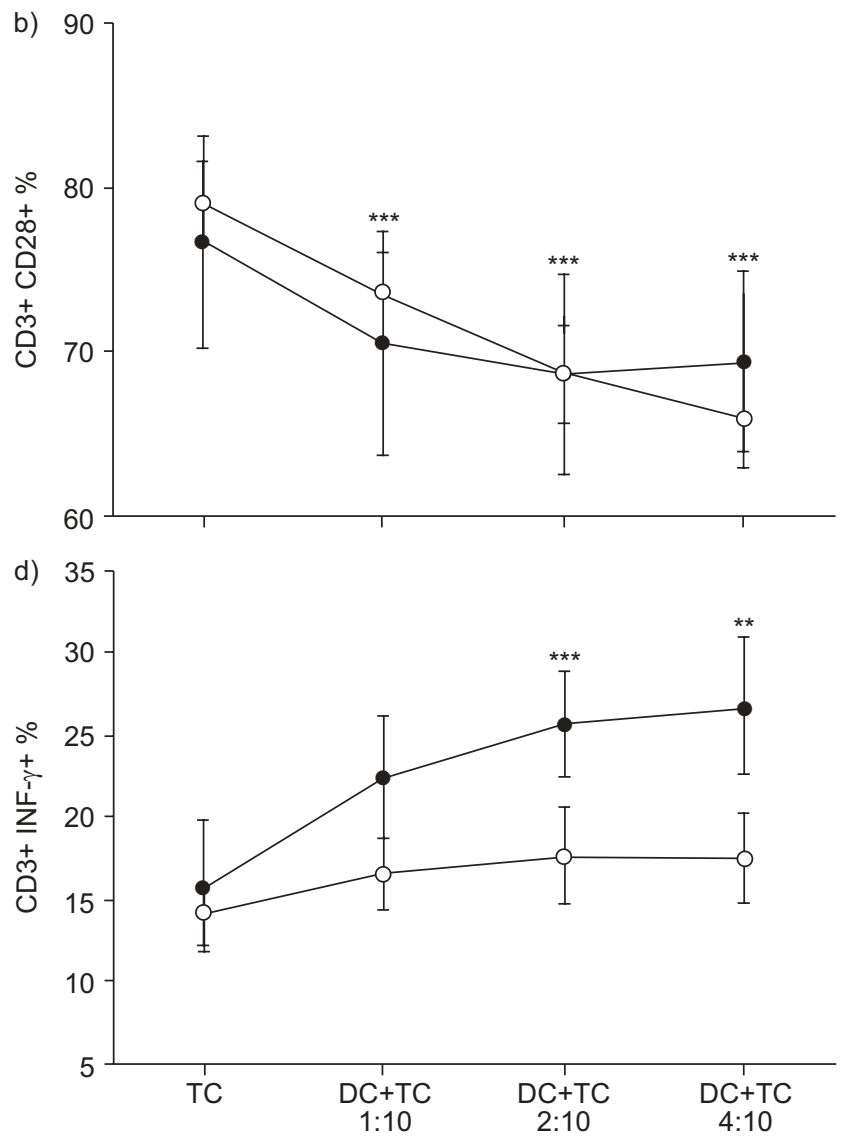

FIGURE 3. T-cell phenotype with dendritic cell (DC)/T-cell (TC) ratio increase. CD69, CD28 expression and interleukin (IL)-13 or interferon (IFN)- $\gamma$ producing TCs were assessed by flow cytometry in DC/TC co-culture while increasing DC/TC ratio from 1:10 to 4:10. Results were given for non-OB $(O, n=16)$ and $\mathrm{OB}$ recipients $(\bullet, n=10)$ and compared in each group with baseline. *: $p<0.05 ; * *: p<0.01 ; * \star *: p<0.001$. 

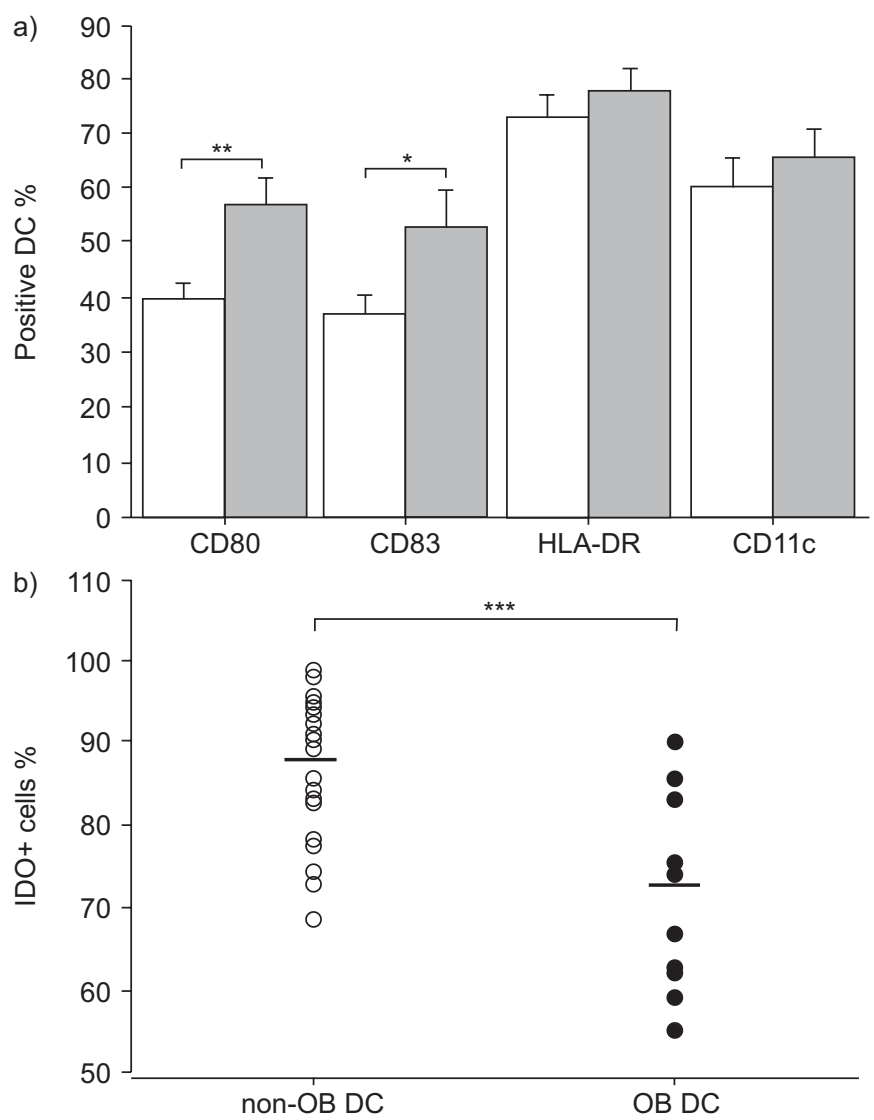

FIGURE 4. Dendritic cell (DC) maturation markers and indoleamine 2,3dioxygenase (IDO) expression. a) CD80, CD83, human leukocyte antigen (HLA)-DR and $\mathrm{CD} 11 \mathrm{c}$ and b) IDO expression by DCs. a) Results were compared between obliterative bronchiolitis (OB; $\square ; n=14)$ and non-OB $(\square ; n=28)$ recipients. b) Horizontal bars show mean and were assessed in DC/T-cell co-culture by flow cytometry. Results were given as percentage of total DC. Results were compared between $\mathrm{OB}(\bullet ; \mathrm{n}=14)$ and non-OB $(\mathrm{O} ; \mathrm{n}=28)$ recipients. *: $\mathrm{p}<0.05$; ${ }^{*}: \mathrm{p}<0.01$; $* * *: p<0.001$

to compare T-cell activation with and without DCs and DC maturation markers expression with or without $P$. aeruginosa or anti-co-receptor Abs. ANOVA was used to test differences between results obtained at various DC/T-cell ratios. In case of significant ANOVA, Wilcoxon signed-rank tests were used to compare each condition versus baseline. A p-value $<0.05$ was considered to be statistically significant for all tests. Results are given as mean $\pm \mathrm{SE}$.

\section{RESULTS}

\section{Patients}

Characteristics of the patients are shown in table 1. A total of 49 consecutive patients ( 24 males, 25 females) who underwent double lung $(n=46)$ or single lung $(n=3)$ transplantation in the present authors' institution (Chest Medicine Dept, Sainte Marguerite University Hospital, Marseille, France) were included in the study. The underlying diagnoses were cystic fibrosis $(n=31)$, emphysema $(n=8)$, bronchiectasis $(n=2)$, pulmonary fibrosis $(n=2)$, primary pulmonary hypertension $(n=2)$, sarcoidosis $(n=1)$, Langerhans cell histiocytosis $(n=1)$, broncholithiasis $(n=1)$ and Rendu-Osler disease $(n=1)$. Of the total, 35 were considered as healthy LTR (mean age $39 \mathrm{yrs}$ ) and 14 displayed BOS (mean age $34.9 \mathrm{yrs}$ ) at the end of the inclusion period. In the present cohort, $\mathrm{OB}$ and non-OB recipient groups showed no significant difference concerning donor/recipient cytomegalovirus status or number of human leukocyte antigen (HLA) mismatches.

\section{DCs from non-OB LTR upregulate T-cell regulatory markers}

The influence of DCs on autologous T-cells in healthy recipients was compared with $\mathrm{OB}$ patients in $\mathrm{DC} / \mathrm{T}$-cell co-cultures. Treg population (CD4+CD25high+Foxp3+) was evaluated after culture as shown in figure 1. In non-OB recipients, DCs induced an increase in Treg cells and IL-10 production (fig. 2). In parallel, T-cell CTLA-4 expression was increased by co-culture whereas ICOS and CD28 were downregulated (fig. 2 and table 2). While T-cell Interferon (IFN)- $\gamma$ production was not affected by DCs, helper T-cell (Th)-2 cytokines significantly decreased (fig. 2 and table 2). In contrast, in OB, neither co-receptor expression nor Th2 cytokines production varied in the presence of DCs (fig. 2 and table 2), while IFN- $\gamma$ was upregulated.

Thus, it appears that DCs from non-OB recipients induced a tolerant phenotype of autologous T-cells while a proinflammatory phenotype is induced in $\mathrm{OB}$ recipients with upregulation of IFN- $\gamma$ production.

\section{The T-cell tolerant phenotype in healthy LTR is linked to the $D C / T$-cell ratio}

In order to assess whether DCs are responsible for the phenotype of T-cells, the DC/T-cell ratio was increased from 1:10 to $4: 10$ in $\mathrm{DC} / \mathrm{T}$-cell co-culture. In non-OB recipients, increase in DC/T-cell ratio induced a deactivation of T-cells, reflected by a downregulation of CD69 and CD28 expression as well as IL-13 production (fig. 3). T-cell activation reached a minimal level at a ratio of 4:10. T-cell IFN- $\gamma$ production did not vary under DC stimulation. By contrast in OB recipients, there was an increase in DC upregulated cytokine production (IL-13, INF- $\gamma$ ) as well as CD69 expression (fig. 3). DC-induced modulation of T-cell activation is thus dose-dependent.

\section{DC from non-OB recipients display an immature phenotype}

The maturation stage of DCs critically impacts the outcome of the immune response. Thus, DC markers related to DC maturation were investigated: CD11c, CD80, CD83, and HLA-DR. Compared with DCs from OB recipients, DCs from non-OB subjects displayed a reduced expression of CD83 and CD80 (fig. 4a), while levels of CD11c and MHC-II were not different.

One mechanism by which APCs may induce a tolerant T-cell response is through the expression of IDO. According to a regulatory phenotype induced upon DC stimulation in non-OB recipients, OB DCs expressed lower levels of intracellular IDO than non-OB patients (fig. 4b).

Thus compared to $\mathrm{OB}$, non-OB DCs exhibit an immature phenotype, despite the use of identical maturating cell culture conditions in both groups. 

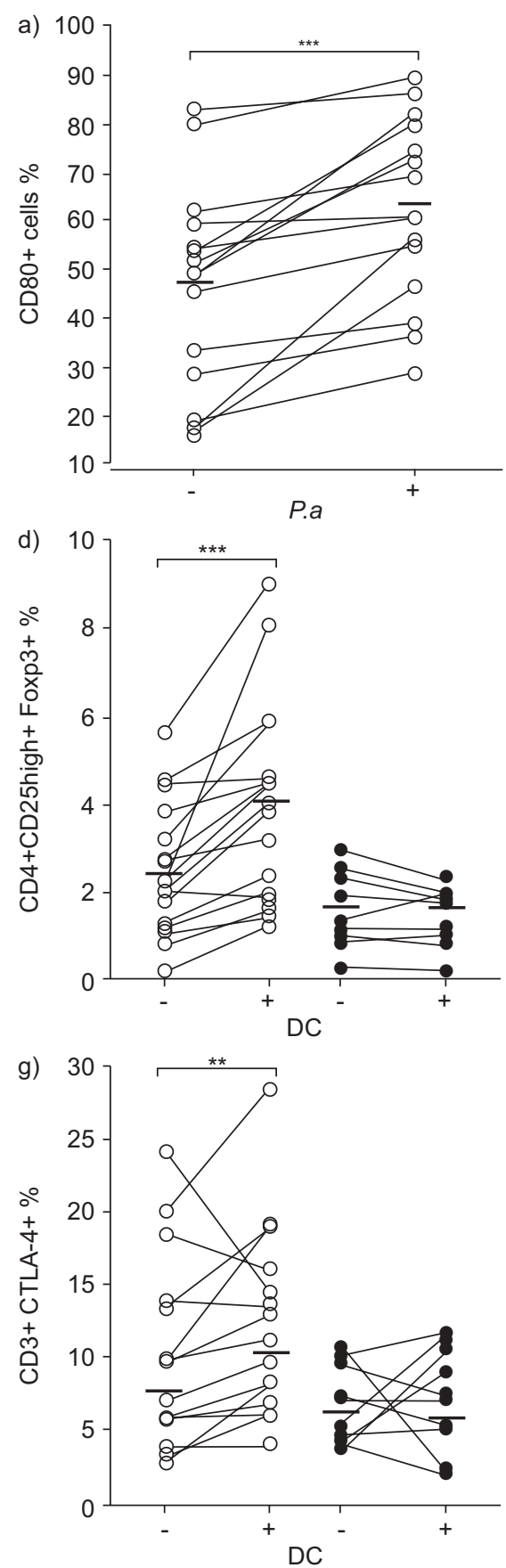
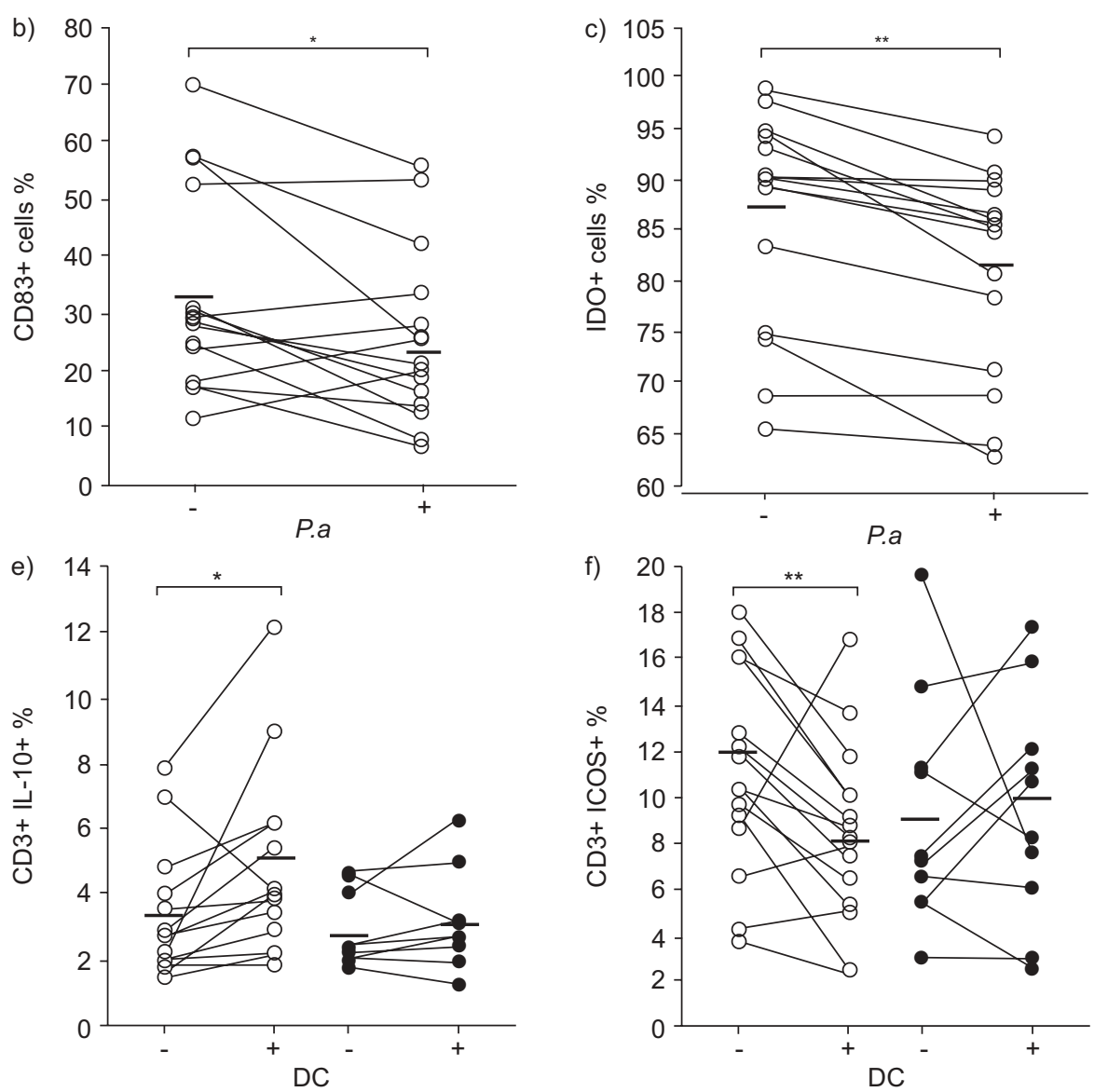

FIGURE 5. T-cell and dendritic cell (DC) phenotype in the presence of Pseudomonas aerugisosa (P.a). DC/Tcell co-cultures where performed in the presence of $P$.a culture supernatant. a) CD80, b) CD83 and c) indoleamine 2,3-dioxygenase (IDO) expression by nonobliterative bronchiolitis DCs $(n=16)$ were assessed by flow cytometry in DC/T-cell co-cultures in the presence (+) or absence (-) of P.a. d) T-regulatory cells (CD4+CD25high+Foxp3+), e) interleukin (IL)-10 producing T-cells, f) inducible T-cell co-stimulator (ICOS) or g) cytotoxic T-lymphocyte antigen (CTLA)-4 expressing T-cells + P.a were also assessed in T-cells from nonobliterative bronchiolitis (non-OB $\mathrm{O} ; \mathrm{n}=20)$ and $\mathrm{OB}$ recipients $(-\mathrm{n}=10)$. Results were compared in the presence $(+)$ or absence $(-)$ of $\mathrm{DCs}$. Horizontal bar shows mean. *: $p<0.05 ;{ }^{* *}: p<0.01$; ${ }^{* *}: p<0.001$.

\section{Stimulation by $\mathrm{P}$. aeruginosa modify DC maturation without affecting their tolerogenic effect}

To verify whether or not the DC-induced tolerant phenotype of T-cell was maintained in the presence of microbial compounds (pathogen-associated molecular patterns and antigens), DC/Tcell co-cultures were stimulated with $10 \mu \mathrm{L}$ of $P$. aeruginosa culture supernatant. Stimulation of non-OB recipient's DCs by $P$. aeruginosa supernatant upregulated DC expression of CD80 and decreased DC intracellular IDO production, with a downregulation of CD83 (fig. 5a). By contrast, in OB DCs, P. aeruginosa induced an increase in CD83 expression without significant variation of IDO or other markers (data not shown).
Thus, stimulation by $P$. aeruginosa modifies the DC maturation state both in non-OB and $\mathrm{OB}$ recipients.

In these conditions, a slight upregulation of T-cell CD69 expression and IFN- $\gamma$ production was induced in OB recipients. Regarding co-receptors and Th2 cytokine production, no significant variation was found (fig. $5 \mathrm{~b}$ and table 3 ) In non-OB recipients, adding DCs in the presence of microbial compounds still upregulated IL-10 production, Treg cells and CTLA-4 expression, whereas CD28 and ICOS expression decreased (fig. 4b and table 3). Regarding Th2 and Th1 cytokines, no significant variation was found (table 3). 


\begin{tabular}{|c|c|c|c|c|}
\hline \multirow[t]{3}{*}{ TABLE 3} & \multicolumn{4}{|c|}{$\begin{array}{l}\text { Effect of dendritic cells on T-cell surface antigen } \\
\text { and cytokine expression in the presence of } \\
\text { Pseudomonas aeruginosa in obliterative } \\
\text { bronchiolitis (OB) and non-OB recipients }\end{array}$} \\
\hline & \multicolumn{2}{|c|}{ non-OB } & \multicolumn{2}{|c|}{ OB } \\
\hline & $-D C$ & $+\mathrm{DC}$ & $-D C$ & $+\mathrm{DC}$ \\
\hline Trials $\mathrm{n}$ & 20 & 20 & 10 & 10 \\
\hline CD3+IL-13+ & $4.12 \pm 0.71$ & $4.46 \pm 0.71$ & $3.80 \pm 0.71$ & $4.14 \pm 0.66$ \\
\hline CD3+IL-4+ & $3.01 \pm 0.41$ & $2.69 \pm 0.28$ & $2.82 \pm 0.64$ & $2.74 \pm 0.58$ \\
\hline CD3+IFN- $\gamma+$ & $10.03 \pm 1.42$ & $12.47 \pm 1.64$ & $16.21 \pm 3.96$ & $20.87 \pm 4.96^{\star}$ \\
\hline CD3+CD69+ & $11.43 \pm 1.16$ & $10.56 \pm 0.96$ & $13.73 \pm 2.23$ & $16.21 \pm 2.65^{*}$ \\
\hline CD3+CD28+ & $82.85 \pm 2.17$ & $76.07 \pm 3.61^{\star \star}$ & $82.16 \pm 3.42$ & $82.34 \pm 3.65$ \\
\hline \multicolumn{5}{|c|}{$\begin{array}{l}\text { Data are presented as mean } \pm \text { SE of total T-cell, unless otherwise stated. T-cells } \\
\text { from } \mathrm{OB} \text { and non-OB recipients were cultured in the presence }(+) \text { or absence }(-) \\
\text { of autologous monocyte-derived dendritic cells }(\mathrm{DC}) \text {, pre-stimulated with } 10 \mu \mathrm{L} \\
\text { of Pseudomonas aeruginosa culture supernatant. Statistics are compared } \\
\text { within each group (non-OB or OB), cytokines and receptors expression in } \\
\text { the presence or absence of DC. IL: interleukin; IFN: interferon. *: } p<0.05 \text {; } \\
\star *: p<0.01 \text {. }\end{array}$} \\
\hline
\end{tabular}

Thus, even in the presence of $P$. aeruginosa, DCs still induced a $\mathrm{T}$-cell tolerant phenotype in non-OB recipients only.

\section{The DC-induced tolerant phenotype of T-cell in non-OB recipients is dependent on CTLA-4}

To test if co-receptor engagement by B7 family molecules was responsible for the DC-induced tolerant profile found in nonOB cells, anti-CD28, anti-ICOS or anti-CTLA-4 blocking Abs were added to DC/T-cell co-cultures.

In non-OB recipients, anti-CTLA-4 Abs decreased IL-10 production and Treg proportion (fig. 6a), whereas anti-CD28 or anti-ICOS Abs had no effect (data not shown).

Furthermore, anti-CTLA-4 Abs dramatically decreased DC CD80 and IDO expression and increased CD83, HLA-DR and CD11c expression by DCs from non-OB recipients (fig. 6b).

By contrast in OB recipients, CTLA- 4 blockade induced an increase in ICOS and CD69 expression (fig. 6a), without significant variation in DC maturation markers (data not shown).

Thus, anti-CTLA-4 Abs reversed the DC-induced T-cell phenotype in non-OB but not in $\mathrm{OB}$ recipients. These results indicate that B7-CTLA-4 interactions in DC/T-cell co-cultures specifically mediate the tolerant phenotype of T-cell induced by DC in non-OB recipients only.

\section{DISCUSSION}

In the present study, the influence of autologous DCs on T-cell activation both in healthy non-OB and $\mathrm{OB}$ recipients was investigated. DCs from non-OB recipients induced an upregulation of IL-10 production and Treg cells. These Treg cells are responsible for tolerance induction in various models of solid organ transplantation [18]. In blood LTR, it has been shown that Treg cells were decreased in BOS recipients compared with healthy recipients [12]. The present findings are concordant with these results, suggesting that DC-induced Treg cells, seen in healthy LTR only, could have a protective role in the transplanted patients. According to a DC-induced tolerant phenotype of T-cells, T-cell CTLA-4 expression increased upon DC stimulation in non-OB recipients. Indeed, CTLA-4 is expressed on activated T-cells and as CD28, binds to CD80 and CD86 [19], but it is a regulatory molecule inhibiting T-cell responses [20].

The in vitro influence of DCs derived from peripheral monocytes on T-cells was examined. Although circulating DC nor pulmonary DC were not studied, the present authors assume that the present results will be relevant in vivo. Indeed, airway's DCs originate in peripheral blood monocytes from the bone marrow $[6,21]$. Monocyte-derived DCs used for in vitro studies have similar co-stimulatory molecules (CD80, CD83) and HLA-DR expression to those from lung DCs [22]. These molecules can be differentially expressed by myeloid DCs and plasmacytoid DCs, the two airways DC subsets able to induce distinct types of immune responses [23]. Analysing DCs from BAL or bronchial biopsies would confirm this analogy, but these cells are not represented enough to be studied properly with regard to their co-stimulatory capacities. Under conditions normally inducing mature DC, an immature phenotype was obtained in non-OB recipients whereas in $\mathrm{OB}$ subjects the expected mature phenotype was achieved. Indeed DCs from non-OB recipients significantly downregulated surface molecules involved in antigen presentation (CD80, CD83) and concomitantly over-expressed intracellular IDO, implicated in inhibition of T-cell proliferation [24]. This immature DC phenotype is likely responsible for the tolerant phenotype of T-cells induced in co-cultures.

$P$. aeruginosa was used to challenge DCs, both by stimulating toll-like receptors (TLR) and by inducing a complete antigen presentation system, since $80 \%$ of the studied patients, as CF or bronchiectasis patients had been previously infected by this pathogen. TLR stimulation by Gram-negative bacteria is expected to induce DCs to differentiate in pro-inflammatory cells able to organise the DC-induced T-cell activation towards a full anti-infectious response. However in the present system, $P$. aeruginosa stimulation had little effect in non-OB patients, whereas it effectively increased the pro-inflammatory T-cell phenotype in $\mathrm{OB}$ patients. This result indicates a robust protolerant profile of DCs in non-OB recipients. It is noteworthy that no difference was seen in patients with pre-transplant $P$. aeruginosa colonisation and other LTR. $P$. aeruginosa stimulation modified the DC phenotype in non-OB recipients notably in increasing CD80 expression and reducing IDO production. However the tolerogenic effect of DCs on T-cells was not modified. This could be related to the fact that supernatant of $P$. aeruginosa culture, and not live bacteria, was used. It is also possible that the 7-day culture was too short to mimic the in vivo effect of $P$. aeruginosa. The present results also indicated that factors, other than the DC's level of maturation, were involved in the tolerogenic process. Indeed engagement of CTLA-4 proved to be pivotal.

The use of recombinant soluble form of human CTLA-4 previously demonstrated that CTLA-4/B7 system may regulate IDO expression in CD4+ and CD8+ T-cells [25]. 

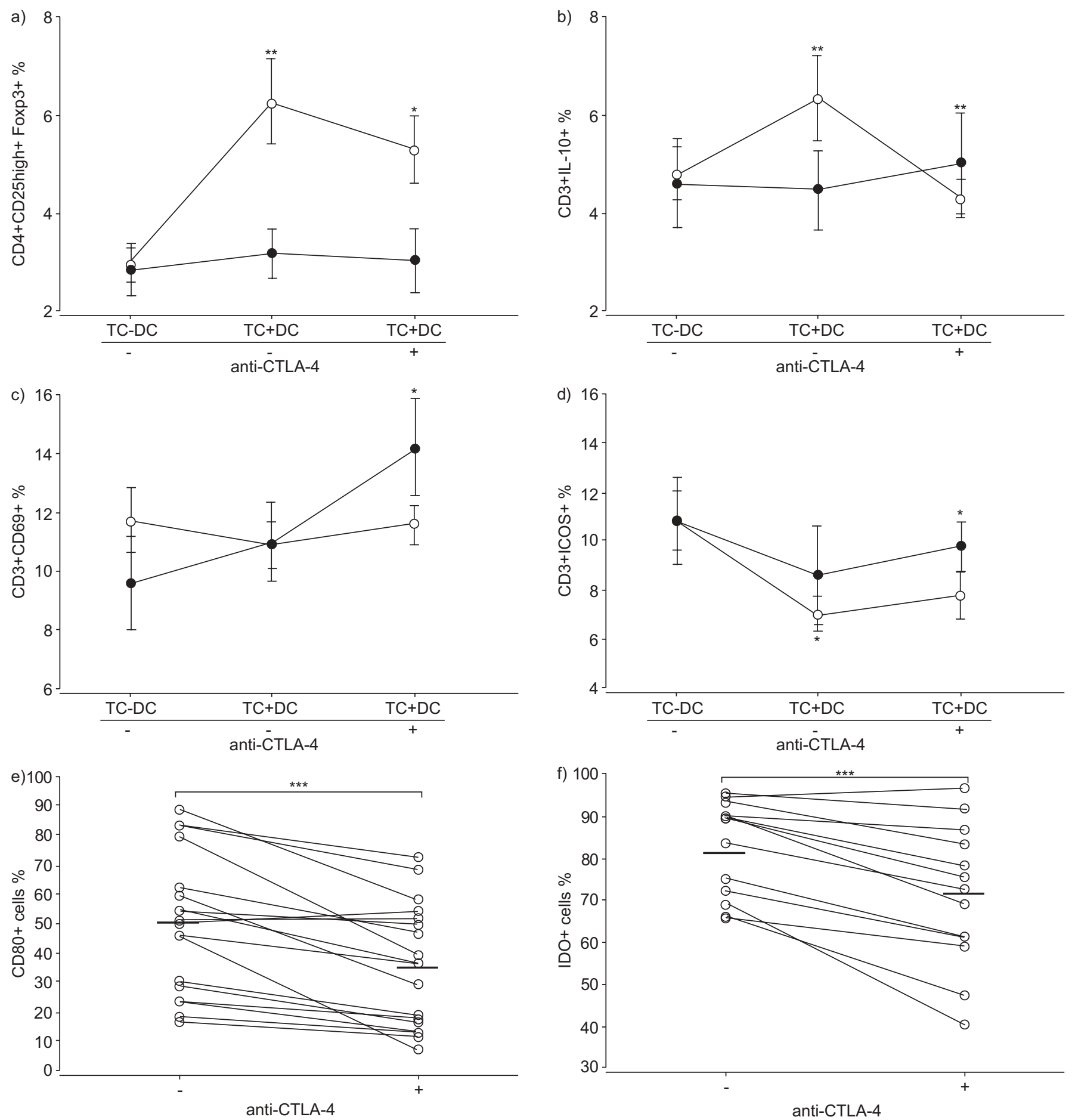

FIGURE 6. T-cell phenotype and dendritic cell (DC) markers expression in the presence of anti-cytotoxic T-lymphocyte antigen (CTLA)-4 antibodies (Abs). a) T-regulatory cells (CD4+CD25high+Foxp3+), b) interleukin (IL)-10 producing T-cells, c) CD69 and d) inducible T-cell co-stimulator (ICOS) expression was assessed by flow cytometry in nonobliterative bronchiolitis (non-OB; $O ; n=18)$ and $\mathrm{OB}$ recipients $(\bullet ; n=10)$. Data are expressed as percentage of total T-cells and compared in the presence $(+)$ or absence (-) of DCs and anti-CTLA-4 Abs. e) CD80 and f) indoleamine 2,3-dioxygenase (IDO) expression by non-OB DCs ( $\mathrm{n}=16$ ) were assessed in DC/T-cell co-culture. Data are given as percentage of total $\mathrm{DC}$ and compared in the presence (+) or absence (-) of anti-CTLA-4 Abs. Horizontal bar shows mean. *: $p<0.05 ;{ }^{* *}: p<0.01 ; * \star *: p<0.001$.

Herein, anti-CTLA-4 Abs induced, in non-OB recipiens, a concomitant decrease of the Treg population and T-cell IL-10 production, demonstrating that the DC-induced tolerant T-cell phenotype observed in this group depends on CTLA-4 engagement $[26,27]$. It is noteworthy that in $\mathrm{OB}$ recipients, CTLA-4 blockade increased T-cell CD69 expression, showing that the receptor is functional but incapable of inducing the tolerant phenotype in these patients. In addition, anti-CTLA-4 
Abs specifically induced a decrease in DC IDO expression and an increased expression of DC membrane antigens which is related to maturation. These results demonstrate that the CTLA-4/B7 axis works not only from DC to T-cell but also from T-cell to DC, in an amplification loop. Nevertheless, anti-CTLA-4 Abs influence neither Th1 nor Th2 cytokine production. In addition to CTLA-4, other co-receptors may be implicated in regulating $\mathrm{T}$-cell responses. Indeed, MUNN et al. [28] showed that blocking CTLA-4 concomitantly to CD28 co-receptor induced a complete abrogation of IDOmediated inhibition on T-cells. In the present study, anti-CD28 had no visible effect alone, but residual effect of DC on T-cells treated by anti-CTLA-4 Abs could still be due to CD28. In addition, a very recent study established a critical role in the control of initiation and reversion of T-cell anergy of a new molecular pathway involving B7-H1 on APCs and its receptor on T-cells' programmed death-1 [29]. It will be interesting to study the B7-H1/programmed death-1 axis in parallel to B7/ CTLA-4 pathway in the present system.

Whether results could have been biased by the use of a different immunosuppression regimen in both groups is questionable. However, the tolerant profile of DC/T-cell interactions found in non-OB recipients was maintained when comparing non-OB and $\mathrm{OB}$ recipients having the same immunosuppressive regimen (tacrolimus, mycophenolate mofetil and azithromycin; data not shown). Furthermore, treatments given in $\mathrm{OB}$ or after acute rejection are more immunosuppressive and therefore expected to increase the Tcell tolerant phenotype, thus diminishing the differences observed between the two groups rather than amplifying them.

As cytokines are major regulators of the immune system, polymorphisms in cytokine and cytokine-receptor genes might influence susceptibility to rejection. LACHA et al. [30] demonstrated that IL-6 gene polymorphism was associated with worse 5-yr outcome in kidney recipients. TLR-4 has also been implicated in allograft rejection notably after cardiac [31] and renal transplantation. Indeed, renal transplant recipients with TLR-4 polymorphism displayed a lower risk of acute allograft rejection [32]. In the same view, the present CTLA-4 results could be the consequence of polymorphism in the CTLA-4 gene. Indeed, it was recently shown that a specific CTLA-4 haplotype (+49A/+6230G), which encodes for normal membrane-bound CTLA-4 expression but reduced soluble CTLA-4 production, was a co-dominant risk allele for acute rejection after clinical liver transplantation [33]. The HLA polymorphism can also be involved. It was shown recently that soluble HLA-G was associated to immunological heart and liver tolerance [34-36].

Taken together, the present results provide new evidence for specific cross-talks between dendritic cells and T-cells in lung transplant recipients according to the presence of obliterative bronchiolitis. The dendritic cell induced tolerant T-cell phenotype observed in nonobliterative bronchiolitis patients provides new insights into the pathophysiology of obliterative bronchiolitis. As dendritic cells are the major cell type presenting antigens in lung and bronchi, the present authors speculate that characteristics of dendritic cell/T-cell interactions induced in nonobliterative bronchiolitis patients are responsible for long-term tolerance. The signals leading to these characteristics are an important topic for future study. Importantly, if this characteristic dendritic cell/T-cell interaction precludes the occurrence of obliterative bronchiolitis, these co-cultures could be instrumental in the early diagnosis of chronic rejection. Finally, the use of autologous dendritic cells has been tested in some cancer therapy to induce a strong anti-tumoural effect [37]. The present results suggest that in a similar approach, immature autologous dendritic cells, Treg cells or a strategy aiming to stimulate the B7/cytotoxic Tlymphocyte antigen- 4 axis could be used to treat or to prevent obliterative bronchiolitis in lung transplant recipients.

\section{REFERENCES}

1 Trulock EP, Edwards LB, Taylor DO, et al. Registry of the International Society for Heart and Lung Transplantation: twenty-third official adult lung and heart-lung transplantation report - 2006. J Heart Lung Transplant 2006; 25: 880-892.

2 van Den Berg JW, Geertsma A, van Der Bij W, et al. Bronchiolitis obliterans syndrome after lung transplantation and health-related quality of life. Am J Respir Crit Care Med 2000; 161: 1937-1941.

3 Yousem SA. Significance of clinically silent untreated mild acute cellular rejection in lung allograft recipients. Hum Pathol 1996; 27: 269-273.

4 Reynaud-Gaubert M. [Pathophysiology of obliterative bronchiolitis in lung transplants]. Rev Mal Respir 2003; 20: 224-232.

5 Jaramillo A, Fernáandez FG, Kuo EY, Trulock EP, Patterson GA, Mohanakumar T. Immune mechanisms in the pathogenesis of bronchiolitis obliterans syndrome after lung transplantation. Pediatr Transplant 2005; 9: 84-93.

$6 \mathrm{McDyer}$ JF. Human and murine obliterative bronchiolitis in transplant. Proc Am Thorac Soc 2007; 4: 37-43.

7 Gimino VJ, Lande JD, Berryman TR, King RA, Hertz MI. Gene expression profiling of bronchoalveolar lavage cells in acute lung rejection. Am J Respir Crit Care Med 2003; 168: 1237-1242.

8 Kelly K, Hertz MI. Obliterative bronchiolitis. Clin Chest Med 1997; 18: 319-338.

9 Merad M, Collin M, Bromberg J. Dendritic cell homeostasis and trafficking in transplantation. Trends Immunol 2007; 28: 353-359.

10 Yousem SA, Ray L, Paradis IL, Dauber JA, Griffith BP. Potential role of dendritic cells in bronchiolitis obliterans in heart-lung transplantation. Ann Thorac Surg 1990; 49: 424-428.

11 Leonard CT, Soccal PM, Singer L, et al. Dendritic cells and macrophages in lung allografts: a role in chronic rejection? Am J Respir Crit Care Med 2000; 161: 1349-1354.

12 Meloni F, Vitulo P, Bianco AM, et al. Regulatory CD4+CD25+ $\mathrm{T}$ cells in the peripheral blood of lung transplant recipients: correlation with transplant outcome. Transplantation 2004; 77: 762-766.

13 Estenne M, Maurer JR, Boehler A, et al. Bronchiolitis obliterans syndrome 2001: an update of the diagnostic criteria. J Heart Lung Transplant 2002; 21: 297-310.

14 Dauer M, Obermaier B, Herten J, et al. Mature dendritic cells derived from human monocytes within 48 hours: 
a novel strategy for dendritic cell differentiation from blood precursors. J Immunol 2003; 170: 4069-4076.

15 Vandenborre K, Delabie J, Boogaerts MA, et al. Human CTLA-4 is expressed in situ on T lymphocytes in germinal centers, in cutaneous graft-versus-host disease, and in Hodgkin's disease. Am J Pathol 1998; 152: 963-973.

16 Mamessier E, Nieves A, Vervloet D, Magnan A. Diesel exhaust particles enhance T-cell activation in severe asthmatics. Allergy 2006; 61: 581-588.

17 Figdor CG, de Vries IJ, Lesterhuis WJ, Melief CJ. Dendritic cell immunotherapy: mapping the way. Nat Med 2004; 10: 475-480.

18 Akl A, Luo S, Wood KJ. Induction of transplantation tolerance-the potential of regulatory $\mathrm{T}$ cells. Transpl Immunol 2005; 14: 225-230.

19 Alegre ML, Frauwirth KA, Thompson CB. T-cell regulation by CD28 and CTLA-4. Nat Rev Immunol 2001; 1: 220-228.

20 Walunas TL, Lenschow DJ, Bakker CY, et al. CTLA-4 can function as a negative regulator of $\mathrm{T}$ cell activation. Immunity 1994; 1: 405-413.

21 McWilliam AS, Nelson DJ, Holt PG. The biology of airway dendritic cells. Immunol Cell Biol 1995; 73: 405-413.

22 Cochand L, Isler P, Songeon F, Nicod LP. Human lung dendritic cells have an immature phenotype with efficient mannose receptors. Am J Respir Cell Mol Biol 1999; 21: 547-554.

23 Masten BJ, Olson GK, Tarleton CA, et al. Characterization of myeloid and plasmacytoid dendritic cells in human lung. J Immunol 2006; 177: 7784-7793.

24 Hwu P, Du MX, Lapointe R, Do M, Taylor MW, Young HA. Indoleamine 2,3-dioxygenase production by human dendritic cells results in the inhibition of $\mathrm{T}$ cell proliferation. J Immunol 2000; 164: 3596-3599.

25 Boasso A, Herbeuval JP, Hardy AW, Winkler C, Shearer GM. Regulation of indoleamine 2,3-dioxygenase and tryptophanyl-tRNA-synthetase by CTLA-4-FC in human CD4+ T cells. Blood 2005; 105: 1574-1581.

26 Eagar TN, Karandikar NJ, Bluestone JA, Miller SD. The role of CTLA-4 in induction and maintenance of peripheral T cell tolerance. Eur J Immunol 2002; 32: 972-981.
27 Vandenborre K, Van Gool SW, Kasran A, Ceuppens JL, Boogaerts MA, Vandenberghe P. Interaction of CTLA-4 (CD152) with CD80 or CD86 inhibits human T-cell activation. Immunology 1999; 98: 413-421.

28 Munn DH, Sharma MD, Mellor AL. Ligation of B7-1/B7-2 by human $\mathrm{CD} 4+\mathrm{T}$ cells triggers indoleamine 2,3dioxygenase activity in dendritic cells. J Immunol 2004; 172: 4100-4110.

29 Tsushima F, Yao S, Shin T, et al. Interaction between B7-H1 and PD-1 determines initiation and reversal of T-cell anergy. Blood 2007; 110: 180-185.

30 Lacha J, Hribova P, Kotsch $\mathrm{K}$, et al. Effect of cytokines and chemokines (TGF- $\beta$, TNF- $\alpha$, IL-6, IL-10, MCP-1, RANTES) gene polymorphisms in kidney recipients on posttransplantation outcome: influence of donor-recipient match. Transplant Proc 2005; 37: 764-766.

31 Methe H, Zimmer E, Grimm C, Nabauer M, Koglin J. Evidence for a role of toll-like receptor 4 in development of chronic allograft rejection after cardiac transplantation. Transplantation 2004; 78: 1324-1331.

32 Ducloux D, Deschamps M, Yannaraki M, et al. Relevance of Toll-like receptor-4 polymorphisms in renal transplantation. Kidney Int 2005; 67: 2454-2461.

33 Perkins JD. The influence of CTLA-4 gene polymorphisms on liver transplant outcomes. Liver Transpl 2006; 12: 1552-1553.

34 Luque J, Torres MI, Aumente MD, et al. sHLA-G levels in the monitoring of immunosuppressive therapy and rejection following heart transplantation. Transpl Immunol 2006; 17: 70-73.

35 Baştürk B, Karakayali F, Emiroğlu R, et al. Human leukocyte antigen-G, a new parameter in the follow-up of liver transplantation. Transplant Proc 2006; 38: 571-574.

36 Le Rond S, Azéma C, Krawice-Radanne I, et al. Evidence to support the role of HLA-G5 in allograft acceptance through induction of immunosuppressive/regulatory $\mathrm{T}$ cells. J Immunol 2006; 176: 3266-3276.

37 de Vries IJ, Lesterhuis WJ, Scharenborg NM, et al. Maturation of dendritic cells is a prerequisite for inducing immune responses in advanced melanoma patients. Clin Cancer Res 2003; 9: 5091-5100. 